\title{
Analgesic effects of piritramide in acute postoperative pain - comparison of intramuscular administration with patient-controlled intravenous analgesia and impact of OPRM1 and $A B C B 1$ polymorphisms
}

\author{
Olga Bartosovaa ${ }^{a}$, Martin Sima ${ }^{a}$, Ondrej Polanecky ${ }^{b}$, Frantisek Perlika, Svatopluk Adamek ${ }^{b}$, Robert Lischke ${ }^{b}$, Ondrej Slanar ${ }^{a}$
}

Aims. The aim of this study was to compare the efficacy, consumption and safety after piritramide administered either intramuscularly (IM) on demand or via patient-controlled intravenous analgesia (PCA) and to examine the impact of $O P R M 1$ and $A B C B 1$ gene polymorphisms on the drug efficacy/safety in both regimens.

Methods. One hundred and four patients scheduled for elective inguinal hernioplasty received piritramide with PCA or IM for postoperative pain management. We evaluated piritramide consumption, pain intensity using visual analogue scale (VAS) and adverse effects.

Results. Median (IQR) piritramide consumption was 18.5 (13.5-31.2) and 15.0 (15.0-15.0) $\mathrm{mg}$ in the PCA and IM groups, respectively $(P=0.0092)$. The respective values of area under the VAS $2-16$-time curve were 40 and $280 \mathrm{~mm}$.h $(P=0.0027)$. Opioid-induced adverse effects were more frequent in the PCA than in the IM group. Variant OPRM1 allele was associated with decreased pain relief, increased opioid consumption and increased incidence of adverse effects, while $A B C B 1$ polymorphisms showed no impact on the observed parameters.

Conclusions. We observed higher piritramide consumption, better pain relief and slightly worse safety profile in the PCA group compared with IM administration. Variant OPRM1 118G allele carriers required higher opioid dosing and suffered from more adverse effects, however, the differences between genotypes have been less pronounced in the PCA patients likely due to improved pain management via PCA.

Key words: analgesia, opioid, piritramide, postoperative pain, adverse effects, gene polymorphism, intramuscular administration, patient-controlled intravenous analgesia

Received: September 6, 2020; Revised: November 4, 2020; Accepted: November 4, 2020; Available online: November, 26, 2020 https://doi.org/10.5507/bp.2020.053

(c) 2022 The Authors; https://creativecommons.org/licenses/by/4.0/

${ }^{a}$ Department of Pharmacology, First Faculty of Medicine, Charles University and General University Hospital in Prague, Albertov 4, 12800 Prague 2, Czech Republic,

${ }^{b}$ Third Department of Surgery, First Faculty of Medicine, Charles University in Prague and Motol University Hospital, V Uvalu 84, 15006 Prague 5, Czech Republic

Corresponding author: Martin Sima, e-mail: martin.sima@lf1.cuni.cz

\section{INTRODUCTION}

Opioids represent key drugs frequently used in postoperative pain management ${ }^{1}$. Their administration via various routes may translate in unequal efficacy or safety profiles of the drugs and it may also affect the drug consumption for achievement of sufficient pain relief ${ }^{2-4}$. Early postoperative total amount of opioid (morphine, meperidine, other) consumption in patients using patient-controlled analgesia (PCA) was substantially higher $(136.9 \pm 100.8 \mathrm{mg})$ compared with subjects receiving intramuscular applications (IM) $(50.8 \pm 34.6 \mathrm{mg})$ in the retrospective study by Everett et al. ${ }^{5}$.

Piritramide is a synthetic opioid for parenteral use with a relative potency of 0.65 to 0.75 in comparison with morphine. It is commonly used for the treatment of postoperative pain and the safety profile of piritramide is comparable to that of morphine ${ }^{6}$. Piritramide is metabolized into inactive compounds in the liver offering a treatment advantage over morphine with active metabolites that may accumulate in patients with decreased renal functions.
Clinical relevance of the opioid receptor $\mu 1$ (OPRM1) gene polymorphism rs 1799971 has been documented for morphine, fentanyl, remifentanil, and sufentanil treatment of acute pain ${ }^{7-9}$. Our previous prospective study further indicated that homozygotes for the variant $\mathrm{G}$ allele benefitted less from piritramide-based postoperative pain treatment than wild-type allele carrying subjects ${ }^{10}$. The sum of pain intensity difference $\left(\right.$ SPID $_{0.6}$ ) was substantially lower (10.0, 20.5, and 22.9 for homozygote, heterozygote, and wild-type homozygote subjects, respectively) although the drug consumption as well as adverse drug reaction occurrence increased significantly in the allele carrying patients. The clinical relevance of genetic polymorphism of an efflux pump involved in transmembrane transport of opioids ABCB1, is still to be established ${ }^{11,12}$.

The aim of this study was to compare the efficacy, safety and consumption of piritramide after IM and PCA administration in patients after elective inguinal hernioplasty. We also aimed to describe the clinical impact of $O P R M 1$ and $A B C B 1$ gene polymorphisms in these two administration routes of piritramide. 


\section{MATERIALS AND METHODS}

\section{Patients and surgical procedure}

This was a randomized, prospective open (laboratory blinded) study in patients undergoing elective inguinal hernioplasty conducted at the $3^{\text {rd }}$ Department of Surgery, First Faculty of Medicine, Charles University in Prague and University Hospital Motol. The study was approved by the local Ethics Committee under the No. EK 611/09. It was conducted in accordance with the Declaration of Helsinki.

Patients were included according to the following criteria: age 18-75 years, diagnosed inguinal hernia, signed informed consent, ability and willingness to use PCA pump. Main exclusion criteria included allergy to opioids, unwillingness to cooperate in the pain assessment, and administration of non-steroidal anti-inflammatory analgesics and/or opioids one week before the surgery. Standard premedication protocol included diazepam $(5 \mathrm{mg})$ per os at the evening before surgery. All patients underwent surgery under standardized general anaesthesia, based on the combination of propofol $(2 \mathrm{mg} / \mathrm{kg})$ and sufentanil $(2 \mu \mathrm{g} / \mathrm{kg})$. Surgical procedures were conducted by an experienced surgeon, who followed standard inguinal hernioplasty according to Lichtenstein.

\section{Postoperative pain treatment and efficacy analysis}

All patients have been randomly assigned to receive piritramide either via PCA or IM route. PCA treated patients were applied drug using Infusomat ${ }^{\circledR}$ Space (Braun, Germany) that was configured to deliver a bolus dose of $0.044 \mathrm{mg} / \mathrm{kg}$ with a $10 \mathrm{~min}$ lock-out period. IM group received piritramide (Dipidolor inj. sol. Janssen-Cilag Ltd) intramuscularly after returning from surgery and then on demand at a fixed dose of $15 \mathrm{mg}$ for one application with the minimal dosing interval of $8 \mathrm{~h}$. Rescue medication was standardized as follows: $1000 \mathrm{mg}$ of dipyrone (Novalgin inj. sol. Sanofi-aventis s.r.o.) administered intravenously, in case of insufficient pain relief a bolus of $5 \mathrm{mg}$ of morphine (Morphine Biotica 1\% inj. sol. BB Pharma, a.s.) was to be administered according to established analgesic protocol.

Patient demographic and clinical characteristics and piritramide dosing data were recorded. Pain intensity was assessed using visual analogue scale (VAS 0-100 mm) at $2,4,6,8,12$ and $16 \mathrm{~h}$ after the surgery. The piritramide consumption was evaluated at the same time. In addition, appearance of opioid-induced adverse effects was recorded. Postoperative nausea and vomiting (PONV) were considered as certain opioid-induced adverse effects, while headache, sedation, pruritus and vertigo represented possible opioid-induced adverse effects. Samples and clinical data were coded and all subsequent analyses were conducted as blinded to any patients" data.

\section{Genotyping}

Peripheral venous blood samples were collected in tubes containing K2EDTA. The samples were immediately frozen and stored at $-20{ }^{\circ} \mathrm{C}$ until further processing. DNA was subsequently isolated using QIAmp Blood Mini Kit (Qiagen, Germany). Genotyping for ABCB 1 (rs 1045642, rs2032582) and OPRM1 (rs 1799971) polymorphisms was done as published previously ${ }^{10,13}$.

\section{Data analysis and statistics}

Sample size calculation was performed prior to commencement of the study. Our previous data on PCA administered piritramide indicated mean $\operatorname{SPID}_{0-16}(\mathrm{SD})$ of $40.33(9.19) \mathrm{mm}\left(\right.$ ref. $\left.^{10}\right)$. To test the significance of differences between the groups of $6.05 \mathrm{~mm}$ ( $15 \%$ of mean), with $90 \%(\beta=0.1)$ power and $\alpha=0.05$ a minimum sample size of 48 patients per group was estimated.

The area under the VAS-time curve from 2 to $16 \mathrm{~h}$ (AUC2-16) was calculated using the trapezoidal rule.

Table 1. Patient characteristics; genotype frequencies of OPRM1 (rs 1799971) and ABCB1 (rs 1045642, rs2032582).

\begin{tabular}{|c|c|c|c|c|}
\hline & & Patient controlled analgesia & Intramuscular injection & $P$ \\
\hline Gender $(\mathrm{M} / \mathrm{F})$ & & $43 / 8$ & $46 / 7$ & 0.7849 \\
\hline Smoking (N/Y) & & $39 / 12$ & $42 / 11$ & 0.8151 \\
\hline Age (years) & & $65(50.5-70)[31-80]$ & $58(44-68)[23-86]$ & 0.1108 \\
\hline Body weight (kg) & & $81(69-89)$ [54-105] & $79(70-85)[38-111]$ & 0.7107 \\
\hline Height (cm) & & $175(168-182)$ [157-190] & $172(167-176)[148-190]$ & 0.0697 \\
\hline $\mathrm{BSA}\left(\mathrm{m}^{2}\right)$ & & $1.93(1.81-2.10)[1.63-2.19]$ & $1.88(1.79-2.04)[1.26-2.33]$ & 0.4376 \\
\hline$O P R M 1$ & $\mathrm{wt} / \mathrm{wt}$ & $38(74.5)$ & $41(77.4)$ & 0.8713 \\
\hline \multirow[t]{2}{*}{$118 A>G$ rs 1799971} & $\mathrm{wt} / \mathrm{v}$ & $10(19.6)$ & $10(18.9)$ & \\
\hline & $\mathrm{v} / \mathrm{v}$ & $3(5.9)$ & $2(3.8)$ & \\
\hline$A B C B 1$ & $\mathrm{wt} / \mathrm{wt}$ & $10(19.6)$ & $12(22.6)$ & 0.0489 \\
\hline \multirow[t]{2}{*}{$3435 C>T$ rs 1045642} & $w t / v$ & $24(47.1)$ & $34(64.2)$ & \\
\hline & $\mathrm{v} / \mathrm{v}$ & $17(33.3)$ & $7(13.2)$ & \\
\hline$A B C B 1$ & $\mathrm{wt} / \mathrm{wt}$ & $16(31.4)$ & $17(32.1)$ & 0.8164 \\
\hline \multirow[t]{2}{*}{$2766 G>T \operatorname{rs} 2032582$} & $\mathrm{wt} / \mathrm{v}$ & $26(51.0)$ & $29(54.7)$ & \\
\hline & $\mathrm{v} / \mathrm{v}$ & $9(17.6)$ & $7(13.2)$ & \\
\hline
\end{tabular}

Data are expressed as median (IQR) [range] or $\mathrm{n}(\%)$

wt, wild-type allele; v, variant allele 
Table 2. Piritramide consumption and analgesic efficacy parameters.

\begin{tabular}{lccc}
\hline & Patient controlled analgesia & Intramuscular injection & $P$ \\
\hline Piritramide consumption $(\mathrm{mg})$ & $18.5(13.5-31.2)[3.6-78.1]$ & $15(15-15)[15-30]$ & 0.0092 \\
VAS score at 2 $\mathrm{h}(\mathrm{mm})$ & $0(0-30)[0-70]$ & $40(20-70)[0-100]$ & $<0.0001$ \\
Maximal VAS score $(\mathrm{mm})$ & $30(0-50)[0-70]$ & $40(30-70)[20-100]$ & $<0.0001$ \\
AUC $_{2-16}$ (mm.h) & $40(0-340)[0-700]$ & $280(180-350)[30-810]$ & 0.0027 \\
\hline
\end{tabular}

Data are expressed as median (IQR) [range]

VAS, visual analogue scale; AUC2-16, area under the VAS-time curve from 2 to $16 \mathrm{~h}$

Table 3. Incidence of opioid-induced adverse effects.

\begin{tabular}{lccc}
\hline & $\begin{array}{c}\text { Patient controlled analgesia } \\
\mathrm{n}(\%)\end{array}$ & $\begin{array}{c}\text { Intramuscular injection } \\
\mathrm{n}(\%)\end{array}$ & $P$ \\
\hline PONV & $9(17.7)$ & $4(7.6)$ & 0.1457 \\
Headache & $4(7.8)$ & $3(5.7)$ & 0.7129 \\
Sedation & $2(3.9)$ & $3(5.7)$ & $>0.9999$ \\
Pruritus & $5(9.8)$ & $1(1.9)$ & 0.1090 \\
Vertigo & $4(7.8)$ & $0(0)$ & 0.0543 \\
Any AE & $12(23.5)$ & $9(17.0)$ & 0.4687 \\
Multiple AEs $(\geq 2)$ & $8(15.7)$ & $2(3.8)$ & 0.0497 \\
\hline
\end{tabular}

PONV, postoperative nausea and vomiting; AE, adverse effect

Table 4. Incidence of opioid-induced adverse effects according to genotype.

\begin{tabular}{|c|c|c|c|c|c|c|c|}
\hline \multirow[t]{3}{*}{ SNP } & \multirow[t]{3}{*}{ Genotype } & \multicolumn{3}{|c|}{$\begin{array}{l}\text { Patient controlled analgesia } \\
\mathrm{n}(\%)\end{array}$} & \multicolumn{3}{|c|}{$\begin{array}{c}\text { Intramuscular injection } \\
\mathrm{n}(\%)\end{array}$} \\
\hline & & AE: yes & AE: no & $P$ & AE: yes & AE: no & $P$ \\
\hline & & & & $\chi^{2}$ & & & $\chi^{2}$ \\
\hline \multirow{3}{*}{$\begin{array}{l}\text { OPRM1 } \\
118 A>G \text { rs } 1799971\end{array}$} & $\mathrm{wt} / \mathrm{wt}$ & $3(8)$ & $35(92)$ & $<0.0001$ & $6(15)$ & $35(85)$ & 0.0058 \\
\hline & $w t / v$ & $6(60)$ & $4(40)$ & & $1(10)$ & $9(90)$ & \\
\hline & $\mathrm{v} / \mathrm{v}$ & $3(100)$ & $0(0)$ & 22.30 & $2(100)$ & $0(0)$ & 10.28 \\
\hline \multirow{3}{*}{$\begin{array}{l}A B C B 1 \\
3435 C>T \text { rs } 1045642\end{array}$} & $\mathrm{wt} / \mathrm{wt}$ & $2(20)$ & $8(80)$ & 0.6636 & $2(17)$ & $10(83)$ & 0.6728 \\
\hline & $w t / v$ & 7 (29) & $17(71)$ & & $5(15)$ & $29(85)$ & \\
\hline & $\mathrm{v} / \mathrm{v}$ & $3(18)$ & $14(82)$ & 0.8200 & $2(29)$ & $5(71)$ & 0.7927 \\
\hline \multirow{3}{*}{$\begin{array}{l}A B C B 1 \\
2766 G>T \text { rs } 2032582\end{array}$} & $\mathrm{wt} / \mathrm{wt}$ & $4(25)$ & $12(75)$ & 0.6815 & $4(24)$ & $13(76)$ & 0.3771 \\
\hline & $\mathrm{wt} / \mathrm{v}$ & $5(19)$ & $21(81)$ & & $5(17)$ & $24(83)$ & \\
\hline & $\mathrm{v} / \mathrm{v}$ & $3(33)$ & $6(67)$ & 0.7670 & $0(0)$ & $7(100)$ & 1.950 \\
\hline
\end{tabular}

SNP, single nucleotide polymorphism; wt, wild-type allele; v, variant allele; AE, adverse effect

Descriptive statistics - median and interquartile range (IQR) were calculated using MS Excel 2010 (Microsoft Corporation, Redmond, USA). For comparison between PCA and IM groups the Mann-Whitney U test and Fisher's exact test were used for continuous and categorical data, respectively. For the evaluation of differences between the genotype groups the Kruskal-Wallis test and $\chi 2$ test were used for the respective data.

GraphPad Prism 8.2.1 software (GraphPad Inc., La Jolla, USA) was used for all comparisons and $P$-levels < 0.05 were considered as statistically significant.

Hardy-Weinberg equilibrium was verified for the observed genotype frequencies of the OPRM1 (rs1799971) and $A B C B 1$ (rs2032582, rs1045642) polymorphisms to detect deviations from the expected genotype distribution.

\section{RESULTS}

In total 104 patients were enrolled, 51 subjects into the PCA group and 53 into the IM group. All recorded demographic data including gender, smoking, age, body weight, height, and body surface area (BSA) were similar in the two groups (Table 1). Median (IQR) length of operation was 47 (36-54) min in the PCA group and it was shorter in comparison with the respective value in the IM group of 52 (45-69) $\min (P=0.0064)$. Anaesthesia length was not different between the two groups with the respective median (IQR) values of 66 (62-72) min and 74 (60-90) $(P=0.0653)$.

Piritramide consumption was significantly higher in the PCA group compared with IM patients. All studied analgesic efficacy parameters have also been significantly different between the two groups (Table 2). Table 3 sum- 

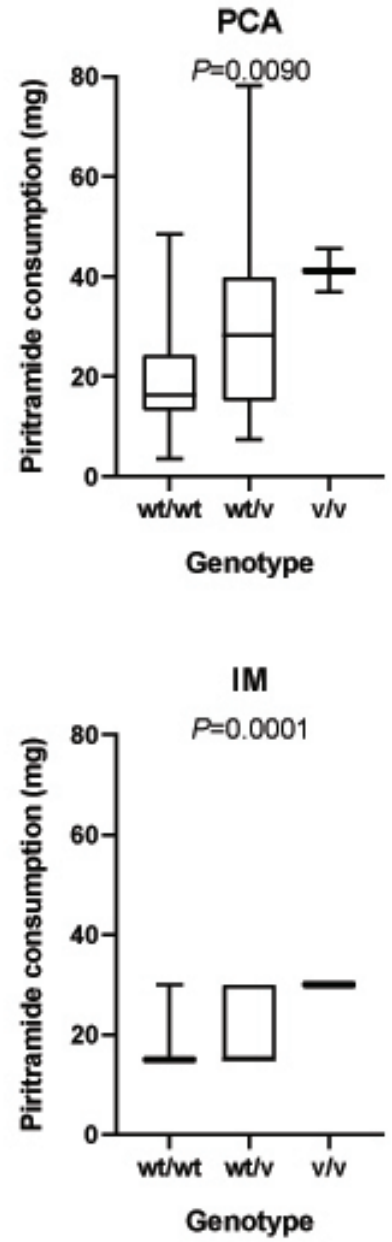
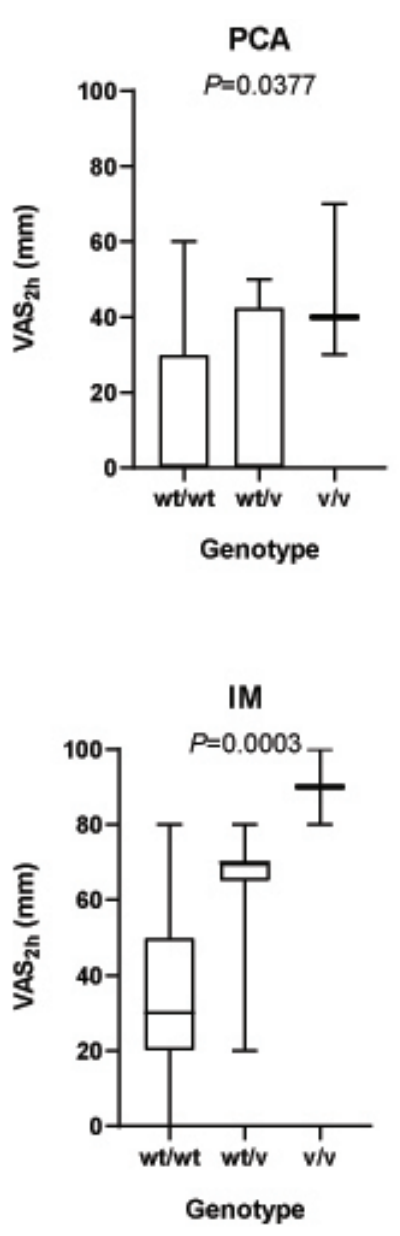
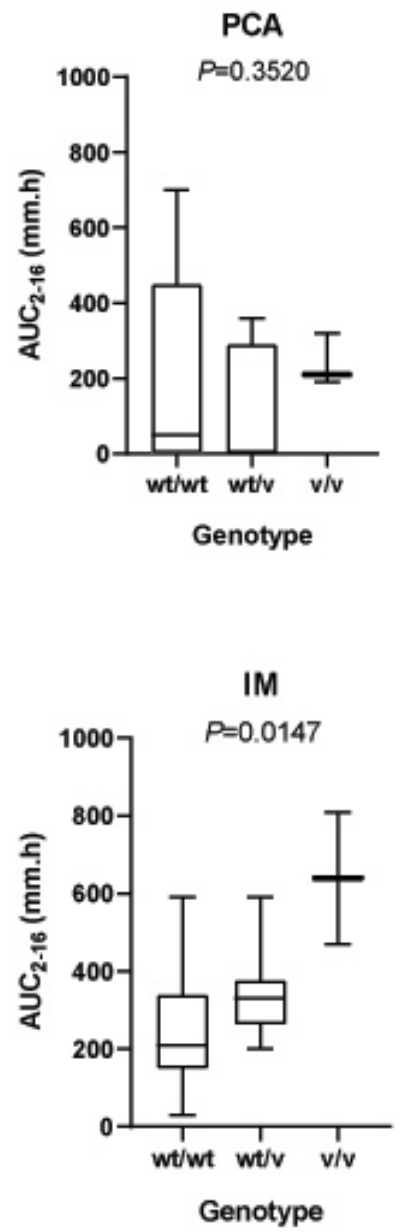

Fig. 1. Impact of OPRM1 (rs1799971) polymorphism on piritramide consumption and analgesic efficacy. PCA, patient controlled analgesia; IM, intramuscular injection; wt, wild-type allele; v, variant allele; VAS, visual analogue scale; $\mathrm{AUC}_{2-16}$, area under the VAS-time curve from 2 to $16 \mathrm{~h}$

marizes the occurrence of opioid-induced adverse effects. The incidence of adverse effects including postoperative nausea and vomiting, headache, sedation, pruritus, and vertigo was not significantly different between the groups. However, higher incidence of multiple adverse effects ( $\geq 2$ adverse effects in one patient) was observed in the PCA group.

Genotype frequencies of OPRM1 (rs 1799971) and ABCB1 (rs 1045642, rs2032582) are presented in Table 1. The impact of OPRM1 (rs 1799971) polymorphism on piritramide consumption and analgesic efficacy is shown in Fig. 1. There was no significant impact of ABCB1 polymorphisms (both rs 1045642 and rs2032582) on piritramide consumption or analgesic efficacy in either study group. An exception was only $\mathrm{AUC}_{2-16}$ that was associated with $\mathrm{ABCB} 1$ (rs 1045642) genotype in the IM group $(P=0.0210)$ with median (IQR) $\mathrm{AUC}_{2-16}$ values of 165 (110-292.5), 295 (210-400), and 160 (140-270) mm.h for wild-type homozygote, heterozygote, and variant homozygote, respectively. Observed opioid-induced adverse effects in genotype subgroups are summarized in Table 4. OPRM1 genotype influenced the incidence of adverse effects, while $\mathrm{ABCB} 1$ genotype showed no significant effect.

\section{DISCUSSION}

We found significantly higher piritramide consumption in the PCA group, which was associated with greater analgesic efficacy (significantly lower $\mathrm{VAS}_{2 \mathrm{~h}}$, maximal VAS, and $\mathrm{AUC}_{2-16} \mathrm{G}$ in comparison with IM administration. However, the higher drug consumption was also accompanied by a clear negative trend in safety profile of PCA. A numerical trend towards higher frequency of postoperative nausea and vomiting, headache, pruritus, and vertigo was seen in the PCA group compared with IM group, which was likely due to the fact that the study was not powered for the safety endpoints. However, significantly higher occurrence of multiple adverse effects has been shown (two or more adverse effects occurred in one patient).

A cochrane review evaluating the efficacy and safety of PCA versus non-PCA (covering 49 studies with 1725 participants on PCA and 1687 participants assigned to a control group) showed that participants receiving PCA had 8.82 (95\% CI: 13.09-4.54) points lower VAS pain intensity scores (from 0 to 100) over 0 to $24 \mathrm{~h}$ versus non-PCA $\left(\right.$ ref. $\left.{ }^{14}\right)$. The mean consumption of intravenous morphine equivalents 0 to $24 \mathrm{~h}$ ranged across control 
groups from 12 to $88 \mathrm{mg}$, while in PCA groups was 7.21 (95\% CI: 1.44-12.98) mg higher. Patients receiving PCA had higher incidence of pruritus ( $15 \%$ versus $8 \% ; P=0.01)$, but the incidence of other adverse events was similar to non-PCA administrations. The results of this study are therefore consistent with the observations seen after other opioids.

The association of OPRM1 rs1799971 polymorphism with pain response was evaluated in a meta-analysis that included 18 studies with a total number of 4607 participants ${ }^{15}$. In the random-effect analysis, variant allele carriers required higher mean opioid dose than wildtype homozygotes (standardized mean difference -0.18 ; $P=0.003$ ). Furthermore, heterozygotes required significantly higher doses of rescue medication and suffered from more sufentanil-induced adverse effects than wildtype homozygotes during the postoperative period ${ }^{16}$. In accordance with these findings we found statistically significant increase in piritramide consumption in the order wild-type homozygotes < heterozygotes < variant homozygotes in both PCA and IM group. The variant homozygotes/wild-type homozygotes piritramide consumption ratio was 2.15 and 1.91 in PCA and IM group, respectively. This finding is in accordance with our previous study, where variant homozygotes/wild-type homozygotes piritramide consumption ratio was 1.91 in PCA patients ${ }^{10}$.

The VAS score at 2 hours was higher in variant allele carriers. The variant homozygote/wild-type homozygote $\mathrm{VAS}_{2 \mathrm{~h}}$ ratio was 4.12 and 2.53 in PCA and IM group, respectively. $\mathrm{AUC}_{2-16}$ was associated with variant allele presence only in IM group, while the $\mathrm{AUC}_{2-16}$ between genotypes were not different in the PCA group. The respective values of the homozygote/wild-type homozygote $\mathrm{AUC}_{2-16}$ ratio in PCA and IM group were 1.17 and 2.52.

Our interpretation of these findings is that the PCA offers better pain management over IM on demand dosing and this advantage of PCA is especially pronounced in difficult-to-treat patients that are likely to respond less to opioids due to the genetic predispositions.

However, higher demand and piritramide consumption in the variant allele carriers was offset by higher occurrence of adverse drug reactions.

Polymorphisms in the $A B C B 1$ gene may alter p-gp expression and activity and thus drug disposition. However, the published results are unclear and have limited clinical relevance ${ }^{17}$. The results from our study also showed no association between genetic polymorphism in the $A B C B 1$ gene and piritramide consumption, analgesic effect or adverse effects, except for a significant difference between $A B C B 1$ rs 1045642 genotypes in $\mathrm{AUC}_{2-16}$ in the IM group. When we analysed this finding, we came to the conclusion that this is most likely an artefact. Firstly, this effect was not seen in the PCA group and secondly, $\mathrm{AUC}_{2-16}$ in IM group increased inconsistently in the order of variant homozygote < wild-type homozygote < heterozygote.

There are a few limitations of the current study. First, since it was carried out in naturalistic clinical settings, the pain intensity was firstly assessed up to $2 \mathrm{~h}$ after surgery. Second, although the study sample size was sufficient for the efficacy endpoint as calculated, the safety profile as well effects in subpopulations e.g. $A B C B I$ haplotype subgroups could not be reliably estimated.

\section{CONCLUSION}

We conclude that patient-controlled intravenous analgesia with piritramide, although associated with higher opioid consumption, leads to better pain relief with slightly worse safety profile in comparison with intramuscular application in the acute postoperative period in patients after elective inguinal hernioplasty. The OPRMI $118 \mathrm{G}$ allele is associated with decreased pain relief after piritramide, which may be partially compensated under PCA settings at the cost of higher drug consumption and increased incidence of adverse effects.

Acknowledgement: The work was supported by the Charles University Project Progres Q25/LF1.

Author contributions: OB, RL, FP, OS: designed the study; OP, SA, RL: gave the clinical and support; OB, OP, SA: performed laboratory diagnostics, collected data; MS: analysed data; OB, MS: manuscript writing (original draft preparation); FP, RL, OS: manuscript writing (review and editing).

Conflict of interest statement: None declared.

\section{REFERENCES}

1. Bethell J, Neuman MD, Bateman BT, Hill AD, Ladha KS, Wijeysundera DN, Wunsch $\mathrm{H}$. Age and postoperative opioid prescriptions: a population-based cohort study of opioid-naive adults. Pharmacoepidemiol Drug Saf 2020;29(4):504-9.

2. Pestano CR, Lindley P, Ding L, Danesi H, Jones JB. Meta-Analysis of the Ease of Care From the Nurses' Perspective Comparing Fentanyl Iontophoretic Transdermal System (ITS) Vs Morphine Intravenous Patient-Controlled Analgesia (IV PCA) in Postoperative Pain Management. J Perianesth Nurs 2017;32(4):329-40.

3. Gulcin Ural S, Yener O, Sahin H, Simsek T, Aydinli B, Ozgok A. The comparison of analgesic effects of various administration methods of diclofenac sodium, transdermal, oral and intramuscular, in early postoperative period in laparoscopic cholecystectomy operations. Pak J Med Sci 2014;30(1):96-100.

4. Katz $P$, Takyar S, Palmer P, Liedgens $H$. Sublingual, transdermal and intravenous patient-controlled analgesia for acute post-operative pain: systematic literature review and mixed treatment comparison. Curr Med Res Opin 2017;33(5):899-910.

5. Everett B, Salamonson Y. Differences in postoperative opioid consumption in patients prescribed patient-controlled analgesia versus intramuscular injection. Pain Manag Nurs 2005;6(4):137-44.

6. Hinrichs $M$, Weyland A, Bantel C. Piritramide : A critical review. Schmerz 2017;31(4):345-52.

7. Bakhouche H, Noskova P, Svetlik S, Bartosova O, Ulrichova J, Kubatova J, Marusicova P, Parizek A, Blaha J, Slanar O. Maternal and neonatal effects of remifentanil in women undergoing cesarean section in relation to $A B C B 1$ and OPRM1 polymorphisms. Physiol Res 2015;64(Suppl 4):S529-38.

8. Hronova K, Pokorna P, Posch L, Slanar O. Sufentanil and midazolam dosing and pharmacogenetic factors in pediatric analgosedation and withdrawal syndrome. Physiol Res 2016;65(Suppl 4):S463-S72.

9. Khalil H, Sereika SM, Dai F, Alexander S, Conley Y, Gruen G, Meng L, Siska P, Tarkin I, Henker R. OPRM1 and COMT Gene-Gene Interaction Is Associated With Postoperative Pain and Opioid Consumption After Orthopedic Trauma. Biol Res Nurs 2017;19(2):170-9. 
10. Bartosova O, Polanecky O, Perlik F, Adamek S, Slanar O. OPRM1 and $A B C B 1$ polymorphisms and their effect on postoperative pain relief with piritramide. Physiol Rese 2015;64(Suppl 4):S521-7.

11. Kim KM, Kim HS, Lim SH, Cheong SH, Choi EJ, Kang H, Choi HR, Jeon JW, Yon JH, Oh M, Shin JG. Effects of genetic polymorphisms of OPRM1, ABCB1, CYP3A4/5 on postoperative fentanyl consumption in Korean gynecologic patients. Int J Clin Pharmacol Ther 2013;51(5):383-92.

12. Zhao Z, Lv B, Zhao X, Zhang Y. Effects of OPRM1 and ABCB1 gene polymorphisms on the analgesic effect and dose of sufentanil after thoracoscopic-assisted radical resection of lung cancer. Biosci Rep 2019;39(1):BSR20181211.

13. Pechandova K, Buzkova H, Slanar O, Perlik F. Polymorphisms of the MDR1 gene in the Czech population. Folia Biol 2006;52(6):184-9.
14. McNicol ED, Ferguson MC, Hudcova J. Patient controlled opioid analgesia versus non-patient controlled opioid analgesia for postoperative pain. Cochrane Database Syst Rev 2015:2015(6):CD003348.

15. Hwang IC, Park JY, Myung SK, Ahn HY, Fukuda K, Liao Q. OPRM1 A118G gene variant and postoperative opioid requirement: a systematic review and meta-analysis. Anesthesiology 2014;121(4):82534.

16. Bartosova O, Polanecky O, Sachl R, Stenglova Netikova I, Perlik F, Adamek S, Lischke R, Slanar O. Epidural analgesia with sufentanil in relation to OPRM1 and ABCB1 polymorphisms. Physiol Res 2019;68(Suppl 1):S59-S64.

17. Wolking S, Schaeffeler E, Lerche H, Schwab M, Nies AT. Impact of Genetic Polymorphisms of ABCB1 (MDR1, P-Glycoprotein) on Drug Disposition and Potential Clinical Implications: Update of the Literature. Clin Pharmacokinet 2015;54(7):709-35. 